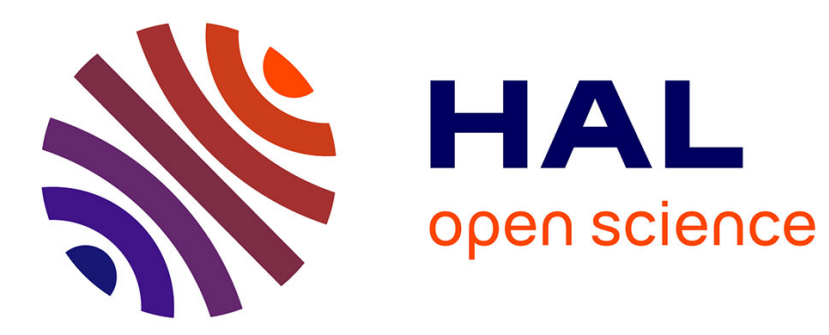

\title{
On the electrochemical impedance response of composite insertion electrodes - Toward a better understanding of porous electrodes
}

David Gruet, Bruno Delobel, David Sicsic, Ivan T. Lucas, Vincent Vivier

\section{- To cite this version:}

David Gruet, Bruno Delobel, David Sicsic, Ivan T. Lucas, Vincent Vivier. On the electrochemical impedance response of composite insertion electrodes - Toward a better understanding of porous electrodes. Electrochimica Acta, 2019, 295, pp.787 - 800. 10.1016/j.electacta.2018.10.115 . hal01922317

\section{HAL Id: hal-01922317 \\ https://hal.science/hal-01922317}

Submitted on 14 Nov 2018

HAL is a multi-disciplinary open access archive for the deposit and dissemination of scientific research documents, whether they are published or not. The documents may come from teaching and research institutions in France or abroad, or from public or private research centers.
L'archive ouverte pluridisciplinaire HAL, est destinée au dépôt et à la diffusion de documents scientifiques de niveau recherche, publiés ou non, émanant des établissements d'enseignement et de recherche français ou étrangers, des laboratoires publics ou privés. 


\title{
On the electrochemical impedance response of composite insertion electrodes - Toward a better understanding of porous electrodes
}

\author{
David GRUET ${ }^{a}$, ${ }^{\text {, Bruno DELOBEL }}$, David SICSIC ${ }^{a}$, Ivan T. LUCAS ${ }^{b}$, Vincent VIVIER ${ }^{b,{ }^{*}}$ \\ ${ }^{a}$ Technocentre Renault, 1 avenue du Golf, 78288 Guyancourt, France \\ ${ }^{b}$ Sorbonne Université, CNRS, Laboratoire Interfaces et Systèmes Electrochimiques, LISE, F-75005 \\ Paris, France
}

\begin{abstract}
Electrochemical Impedance Spectroscopy (EIS) is a powerful tool to assess and understand the degradation of Li-ion battery (LIB) performances during operation (i.e. upon charge and discharge cycles). The full interpretation of EIS diagrams associated to complex electrode/electrolyte interfaces is however tedious. An analytical solution for the electrochemical impedance of a porous electrode composed of spherical intercalation particles in contact with concentrated electrolyte is presented herein. The proposed model which is based on the concentrated solution theory previously developed but which also accounts for kinetic limitations, and both solutionphase and solid-phase diffusion limitations, can predict the EIS response of a porous electrode at any state of charge using a limited set of parameters. Moreover, the developed model can predict the optimal porosity of the electrode to be targeted during the manufacturing process for the best cycling performances depending on the composition of the electrode (volume fractions of active material, binder and conductive agent).
\end{abstract}

Key-Words: Electrochemical impedance spectroscopy; Modeling; Single particle; Porous electrode; Lithium-ion batteries.

*Corresponding author: vincent.vivier@sorbonne-universite.fr 


\section{Introduction}

Insertion materials have been subject of numerous investigations as battery electrode materials, especially alkali and divalent-ion insertion structures [1-5]. Host materials for lithium insertion have been highly optimized and are already widely used in Lithium-ion batteries (LIBs) to power both small electrical devices and electrical vehicles (EV) or hybrid electrical vehicles (HEV). Commercial LIBs are complex systems composed of two porous composite electrodes impregnated with electrolyte. Thus, the understanding of the performance degradation of such systems (specific capacity, charging rate) and their optimization requires the development of non-invasive characterization tools which can be implemented under the conditions of their operation. This can be achieved using electrochemical impedance spectroscopy (EIS) which has become increasingly popular in the LIB community these last decades for the in situ assessment of LIB performances [6-8], since it reflects the kinetics of the processes at the electrodes (charge transfer reactions at the electrode/electrolyte interface and diffusion processes in both solid and liquid phases). The full interpretation of EIS spectra of LIBs can be however tedious because of the complexity of porous composite electrodes made of active material, conductive agent and binder deposited on thin current collectors. Moreover, in a compact battery design, where a third reference electrode cannot be easily introduced, the convoluted impedance response resulting from the contributions of both the positive and the negative electrodes is hardly decipherable. Models can thus be helpful to interpret the behavior of such systems. Most of the time, electrical equivalent circuits (ECCS) are used to describe the electrode/electrolyte interface and to extract parameters (capacitances and resistances) through fittings of EIS spectra $[9,10]$. These empirical models often fail in extracting reliable properties because of the inaccurate description of the electrochemical system under scrutiny. De Levie devised the transmission line model (TLM) [11], in which a first improvement of ECCs was proposed by considering a porous electrode composed of cylindrical pores of finite or infinite length filled with electrolyte. Inspired from de Levie's work, impedance models taking into consideration potential gradient [12], concentration gradient [13] or both [14] also emerged. Later on, impedance models including the mathematical description of the physical processes occurring inside porous electrodes have been developed. In 2000, Meyers et al. [15] established an analytical solution for a single lithium host particle considering 
the kinetics of the electron transfer at the particle/electrolyte interface with the use of the ButlerVolmer equation, and also the kinetics of the lithium diffusion in the particle (i.e. in the solid phase). Assuming a continuous particle-size distribution, they were able to determine the impedance of a porous electrode. Doyle et al. [16] developed a numerical approach to calculate the impedance of a porous electrode system (anode/separator/cathode) taking into account the solution and solid-phase diffusion processes and the interfacial kinetics. Subramanian et al. [17] proposed an analytical solution for a porous electrode by neglecting the solid-phase diffusion process, which was taken into account later on in a model proposed by Sikha and White [18]. They extended this model to an anode/separator/cathode geometry with two porous electrodes [19]. More recently, Huang et al. [20] developed the so-called secondary particle model, which could reproduce the experimental impedance data obtained by Dokko et al. on $\mathrm{LiCOO}_{2}$ particles [21] with a better accuracy than Meyers. They also studied the influence of a passivation layer at the surface of the lithium host particles on the impedance response, and proposed an analytical solution for the impedance of the electrolyte/electrode interface (EEI) [22]. They first analyzed the impedance response by coupling or decoupling faradaic and double layer charging currents and then investigated the impedance response according to the location of the charge transfer reaction at the interface. In 2016, Huang and Zhang [23] proposed an analytical solution of the impedance for three different systems: a blocking electrode, an electrode with faradaic reactions and a porous electrode composed of particles with insertion reactions.

In the present work, we have developed an analytical solution for the potential-dependent current response of a porous composite electrode composed of particles subjected to insertion reactions by considering the kinetics of the electron transfer reactions and the diffusion processes in both solid and liquid phases. The model presented here not only allows to predict the impedance of a porous electrode depending on its state of charge but it is also valid for the whole intercalation range with the same set of parameters. Then, the influence of geometric parameters of the porous electrode on the impedance response was investigated in order to determine the optimal porosity of the electrode associated to the highest performances of the battery. 


\section{Model Development}

When performing EIS, a small amplitude potential or current perturbation (usually a sine-wave) at different frequencies is superimposed to a DC-steady-state potential. The resulting current or potential allows the calculation of a transfer function of the electrochemical system, which in turn allows the EIS spectra to be obtained.

Let us first assume that the amplitude of the perturbation is small enough to consider the system as linear. Thus, each variable of the system can be expressed as a sum of a steady-state contribution and second term corresponding to its response to the perturbation signal at the pulsation $\omega$. For instance, if $X$ is a dependent system variable, this response can be written as

$$
X=\bar{X}+R e[\tilde{X} \cdot \exp (j \omega t)]
$$

where $\bar{X}$ is the steady-state term and $\tilde{X}$ denotes a complex variable.

Interestingly, the linearity condition which is one of the main requirement for performing an EIS measurement usually allows an easy resolution of partial differential equations, thus giving rise to analytical solutions to complex systems describing the overall behavior of the system.

Thus, the first order Taylor expansion allows to express the equations of the system as

$$
f(X)=f(\bar{X})+\frac{\partial f}{\partial X} \operatorname{Re}[\tilde{X} \cdot \exp (j \omega t)]
$$

In the following, an analytical expression of the impedance response for a single intercalation particle is first proposed and then expanded to a porous-electrode system. Although this model describes the insertion in positive or negative porous electrodes, the case of lithium insertion into graphite is addressed in the following.

Using a simple geometry consisting of a spherical insertion particle, we hypothesize that the lithium ion reduction takes place at the surface of the particle only and that the transport of lithium-ion within the particle is achieved only by diffusion, so that the faradaic and non-faradaic contributions in the measured currents can be separated. 
The faradaic current density, corresponding to the charge transfer reaction occurring at the surface of the particle, is described by the Butler-Volmer relation

$$
i_{n}=i_{0}\left[\exp \left(\frac{\alpha_{a} F}{R T}(\Phi-\mathrm{U})\right)-\exp \left(-\frac{\alpha_{c} F}{R T}(\Phi-\mathrm{U})\right)\right]
$$

where $\Phi$ is the potential of the electrode ( $\Phi$ is expressed as the potential difference between the particle, $\varphi_{1}$, and the solution, $\left.\varphi_{2}\right), i_{0}$ is the exchange current density, $\alpha_{a}$ and $\alpha_{c}$ are the charge transfer coefficients assumed equals to $0.5, F$ is the Faraday constant, $R$ is the universal gas constant, $T$ is the temperature and $U$ is introduced as the open-circuit potential of the insertion materials which varies with the concentration of lithium inside the particle [15, 24, 25].

$$
U=U_{\text {stand }}+f\left(c_{s}\right)
$$

$U_{\text {stand }}$ is a standard redox potential and $f\left(c_{S}\right)$ is a function of the amount of lithium inserted in the solid matrix.

Taking advantage of the approximation $\exp (a x) \approx 1+a x$ when $x \rightarrow 0$, the linearization of Butler-Volmer equation expresses as

$$
\tilde{\imath}_{n}=i_{0} \frac{F}{R T}[\widetilde{\Phi}-\widetilde{U}]
$$

Additionally, to account for the surface concentration at the particle, the slope of the titration curve is introduced as

$$
\widetilde{U}=\frac{\partial U}{\partial c_{s}} \tilde{c}_{s}^{\text {surf }}
$$

where $c_{S}$ is the average concentration inside the solid active particle and $\tilde{c}_{S}^{\text {surf }}$ is the surface concentration of the particle. Thus, Eq. 5 can be rewritten as 


$$
\tilde{l}_{n}=i_{0} \frac{F}{R T}\left[\widetilde{\Phi}-\frac{\partial U}{\partial c_{s}} \tilde{c}_{s}^{\text {surf }}\right]
$$

The variation of the concentration within spherical particles of radius $R_{S}$ is given by the second Fick's law, which expresses in spherical coordinates as

$$
\frac{\partial c_{s}}{\partial t}=\frac{D_{s}}{r^{2}} \frac{\partial}{\partial x}\left(r^{2} \frac{\partial c_{s}}{\partial r}\right)
$$

where $r$ is the radial coordinate, $c_{S}$ the surface concentration of the active material particle and $D_{s}$ the diffusion coefficient in the solid phase. The boundary conditions for Eq. 8 are given by:

- $\quad$ at the surface of the particle $\left(r=R_{S}\right)$

$$
i_{n}=-F D_{s} \frac{\partial c_{s}}{\partial r}
$$

- $\quad$ at the center of the particle $(r=0)$

$$
\frac{\partial c_{s}}{\partial r}=0
$$

Using the Laplace transform to express Eq. 8 in the frequency domain, it comes:

$$
j \omega \tilde{c}_{s}=\frac{D_{s}}{r^{2}} \frac{\partial}{\partial x}\left(r^{2} \frac{\partial \tilde{c}_{s}}{\partial r}\right)
$$

The solution of Eq. 11 allows the surface concentration for the particle to be obtained as

$$
\tilde{c}_{s}^{\text {surf }}=\tilde{c}_{s}\left(r=R_{s}\right)=\tilde{\imath}_{n} Y_{s}
$$

with

$$
Y_{s}=\frac{R_{s}}{F D_{s}} \frac{\sinh \left(\sqrt{j \Omega_{s}}\right)}{\sinh \left(\sqrt{j \Omega_{s}}\right)-\sqrt{j \Omega_{s}} \cosh \left(\sqrt{j \Omega_{s}}\right)}
$$


and

$$
\Omega_{s}=\frac{\omega R_{s}^{2}}{D_{s}}
$$

The total current density at the interface between the particle and the solution is given by

$$
\tilde{l}_{\text {total }}=\tilde{l}_{n}+\tilde{l}_{\text {non-faradaic }}
$$

The non-faradaic current density is expressed by the charge and discharge of the double layer as

$$
\tilde{l}_{\text {non-faradaic }}=j \omega C_{d l} \widetilde{\Phi}
$$

where $C_{d l}$ is the double layer capacitance.

Combining equations Eq. 7, Eq. 12, Eq. 13 and Eq. 14, it comes

$$
\tilde{l}_{\text {total }}=\frac{1}{R_{c t}+Y_{s} \frac{\partial U}{\partial c_{s}}} \widetilde{\Phi}+j \omega C_{d l} \widetilde{\Phi}
$$

where the charge transfer resistance $R_{c t}$ is given by

$$
R_{c t}=\frac{R T}{i_{0} F}
$$

The electrochemical impedance of the single particle is given by

$$
Z_{s p}=\frac{\tilde{l}_{\text {total }}}{\widetilde{\Phi}}
$$

that is

$$
Z_{s p}=\frac{1}{\frac{1}{R_{c t}+Y_{s} \frac{\partial U}{\partial c_{s}}}+j \omega C_{d l}}
$$


In a second step, the model was further developed to account for a porous electrode system, i.e. an electrode made of several single particles, as illustrated by the Fig. 1 . The porous electrode model is based on the concentrated solution theory. We consider a 1:1 binary electrolyte (e.g. $\mathrm{Li}^{+}$ and $\mathrm{PF}_{6}{ }^{-}$) with the solvent and we assume a one-dimensional transport inside the electrolyte. Moreover, $D_{e, e f f}$ the effective diffusion coefficient of the electrolyte, $t_{+}$the transference number of lithium ions, $\varepsilon_{e}$ the volume fraction of electrolyte within the electrode (i.e. porosity) and $C_{d l}$ are assumed to be constant, $f_{\mp}$ the mean activity coefficient of the LiPF $_{6}$ solute is supposed constant and equal to 1 .

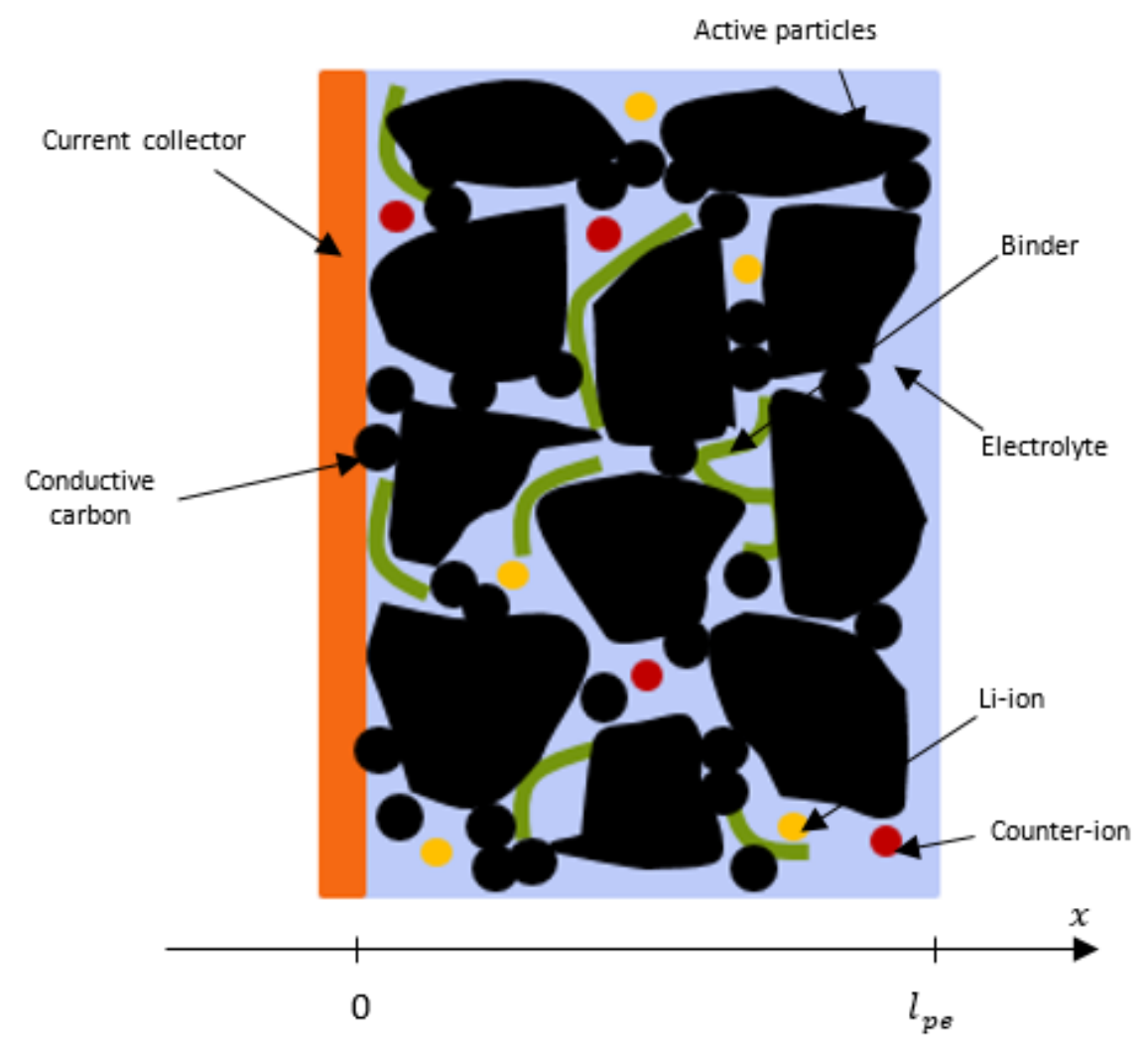

Figure 1: Schematic representation of a typical porous composite electrode (binder, conductive agent and active material, i.e. graphite) in contact with the electrolyte.

The porous electrode model used in this study is derived from a model developed by Huang and Zhang [23] to obtain an analytical solution for the impedance of a porous electrode. The different phenomena taking place in a porous electrode were described using the governing equations of Newman's model [26]. 
The mass balance in the electrolyte expresses as

$$
\varepsilon_{e} \frac{\partial c_{e}}{\partial t}=D_{e, e f f} \frac{\partial^{2} c_{e}}{\partial x^{2}}+\frac{a_{v} i_{l o c}}{z_{+} F}\left(1-t_{+}\right)
$$

where $c_{e}$ is the electrolyte concentration, $i_{l o c}$ the local current density at the electrode/electrolyte interface, $z_{+}$the charge number of cations in the electrolyte, here $z_{+}=1$, and $a_{v}$ the specific surface area equals to $3 \frac{\varepsilon_{a}}{R_{s}}$ for spherical particles where $\varepsilon_{a}$ is the volume fraction of solid active particles.

The charge conservation in the electrolyte phase is given by

$$
i_{2}=-\kappa_{e, e f f} \frac{\partial \varphi_{2}}{\partial x}+\frac{2 \kappa_{e, e f f} R T}{F}\left(1-t_{+}\right) \frac{\partial \ln \left(c_{e}\right)}{\partial x}
$$

where $i_{2}$ is the current density of the electrolyte, $\varphi_{2}$ the potential of the electrolyte and $\kappa_{e, e f f}$ is the effective conductivity of the electrolyte.

The current density of the solid matrix is governed by the Ohm's law

$$
i_{1}=-\kappa_{s, e f f} \frac{\partial \varphi_{1}}{\partial x}
$$

where $i_{1}$ is the current density of the solid matrix, $\varphi_{1}$ is the potential of the electrode and $\kappa_{s, e f f}$ is the effective conductivity of the electrode (under the assumption of an homogeneous conductivity of the porous electrode).

Additionally, the electroneutrality expresses as

$$
\frac{\partial i_{1}}{\partial x}+\frac{\partial i_{2}}{\partial x}=0
$$

The current density of the electrolyte can be expressed as a function of the faradaic current as 


$$
\frac{\partial i_{2}}{\partial x}=a_{v} i_{l o c}
$$

From Eq.21 and Eq.22, we can rewrite Eq. 18, Eq. 19 and Eq. 20 in the frequency domain

$$
\begin{gathered}
j \omega \varepsilon_{e} \tilde{c}_{e}=D_{e, e f f} \frac{\partial^{2} \tilde{c}_{e}}{\partial x^{2}}+\frac{a_{v} \tilde{l}_{l o c}}{F}\left(1-t_{+}\right) \\
a_{v} \tilde{l}_{l o c}=-\kappa_{e, e f f} \frac{\partial^{2} \tilde{\varphi}_{2}}{\partial x^{2}}+\frac{2 \kappa_{e, e f f} R T}{F \bar{c}_{e}}\left(1-t_{+}\right) \frac{\partial^{2} \tilde{c}_{e}}{\partial x^{2}} \\
-a_{v} \tilde{l}_{l o c}=-\kappa_{s, e f f} \frac{\partial^{2} \tilde{\varphi}_{1}}{\partial x^{2}}
\end{gathered}
$$

The local current density is related to the impedance of a single particle $Z_{s p}$ by

$$
\tilde{\varphi}_{1}-\tilde{\varphi}_{2}=Z_{s p} \tilde{l}_{l o c}
$$

Assuming that $Z_{s p}$ is uniform across the porous electrode, the analytical solution for the electrochemical impedance of the porous electrode is given by [23]

$$
\begin{aligned}
Z_{p e}=\frac{l_{p}}{\kappa_{s, e f f}}+ & \frac{a_{v}}{Z_{s p} \kappa_{s, e f f} \tilde{l}_{a p p}}\left(\left(\frac{\alpha_{1}}{\sqrt{\lambda_{1}}}+\frac{\beta_{1}}{\sqrt{\lambda_{2}}}\right) l_{p}+\left(\frac{\alpha_{2}}{\lambda_{1}}+\frac{\beta_{2}}{\lambda_{2}}\right)\right. \\
& \left.-\left(\frac{1}{\lambda_{2}}+\frac{v_{2}}{v_{1}}\right) \Lambda\right)+\frac{1}{\tilde{l}_{a p p}}\left(1-\frac{v_{2}}{v_{1}}\right) \Lambda
\end{aligned}
$$

where $l_{p}$ is the length of the pore and the parameters $\alpha_{1}, \beta_{1}, \alpha_{2}, \beta_{2}, \lambda_{1}, \lambda_{2}, v_{1}, v_{2}$ and $\Lambda$ are parameters whose expressions are given in Appendix 1. 


\section{Results and Discussions}

The connection between the State of Charge $(\mathrm{SoC})$ and the equations of the model is first presented. Then, the results for a single active material particle are presented and the results for a porous electrode are thoroughly discussed in a last part.

- Relation between the SoC, $\frac{\partial U}{\partial c_{S}}$ and $i_{0}$

In this section, we propose to model the impedance spectra at a given SoC of the electrode

material through the use of the two parameters $\frac{\partial U}{\partial c_{s}}$ and $i_{0}$ which depend on the lithium concentration within the host material.

The exchange current density $i_{0}$ can be derived from the Butler-Volmer equation and be expressed as:

$$
i_{0}=F k c_{e}^{\alpha_{a}}\left(c_{t}-C_{S}^{\text {surf }}\right)^{\alpha_{a}}\left(C_{S}^{\text {surf }}\right)^{\alpha_{\iota}}
$$

where $k$ is the electrochemical reaction rate constant in $m . s^{-1}, C_{S}^{\text {surf }}$ is the concentration of Liion at the surface of the particle and $c_{t}$ is the maximum concentration of lithium inside the particle.

To estimate the SoC of the particle, one can consider the lithium insertion ratio, $x$, defined as:

$$
x=\frac{C_{S}^{\text {surf }}}{c_{t}}
$$

Thus, the exchange current density can be rewritten as

$$
i_{0}=F k^{\prime} c_{e}^{\alpha_{a}}(1-x)^{\alpha_{a}} x^{\alpha_{c}}
$$

where $k^{\prime}=k c_{t}$ and expresses in mol. $m^{-2} \cdot s^{-1}$

This relation shows that the exchange current density can be directly linked to the SoC of the porous electrode as shown on Fig. 2a, for different set of parameters $\alpha_{a}$ and $\alpha_{c}$. The other parameters used to calculate $i_{0}$ are reported in Table I. In other words, the knowledge of the 
insertion ratio of the porous electrode allows the kinetic of the charge transfer reaction to be determined.

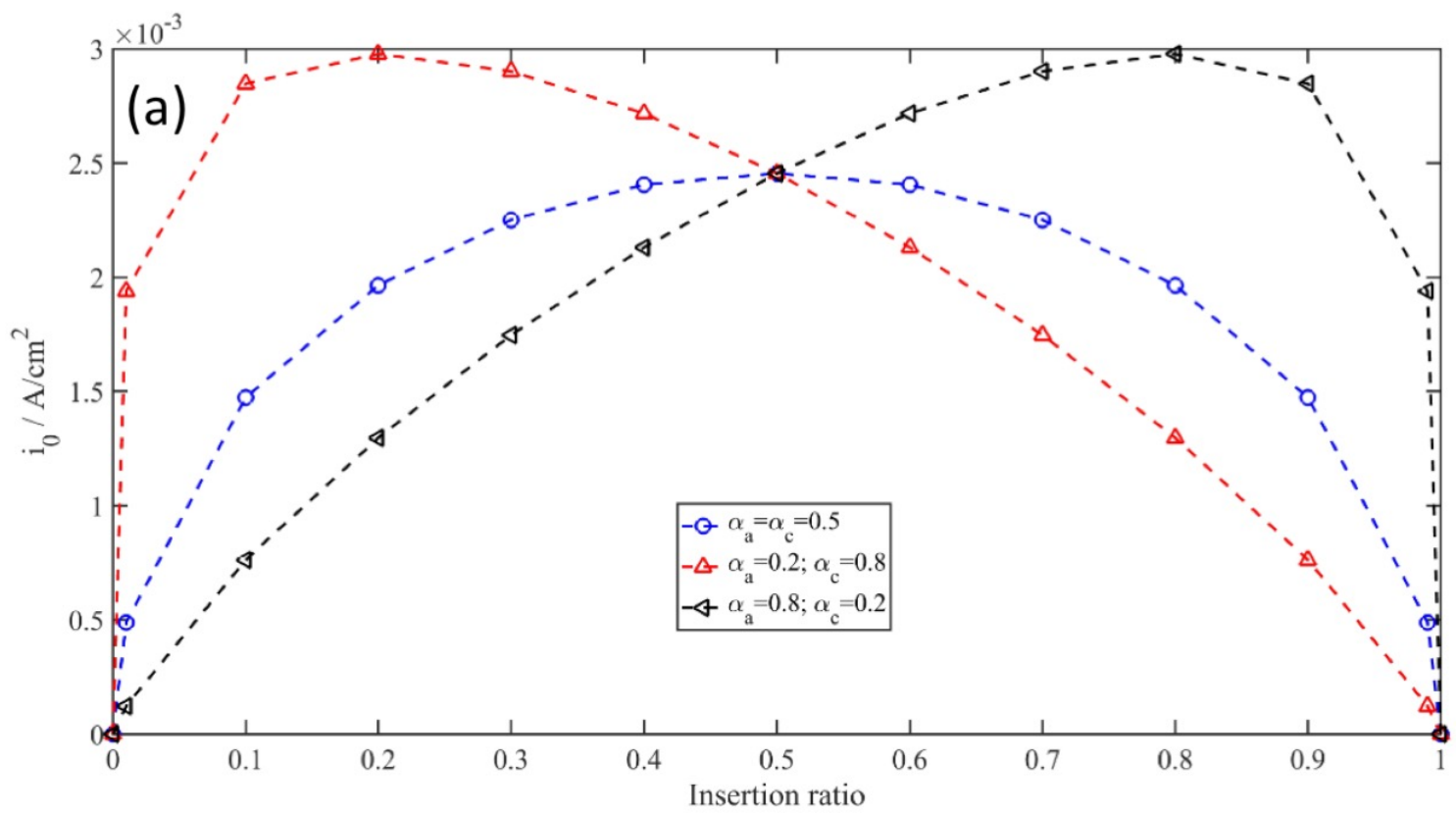

(b)

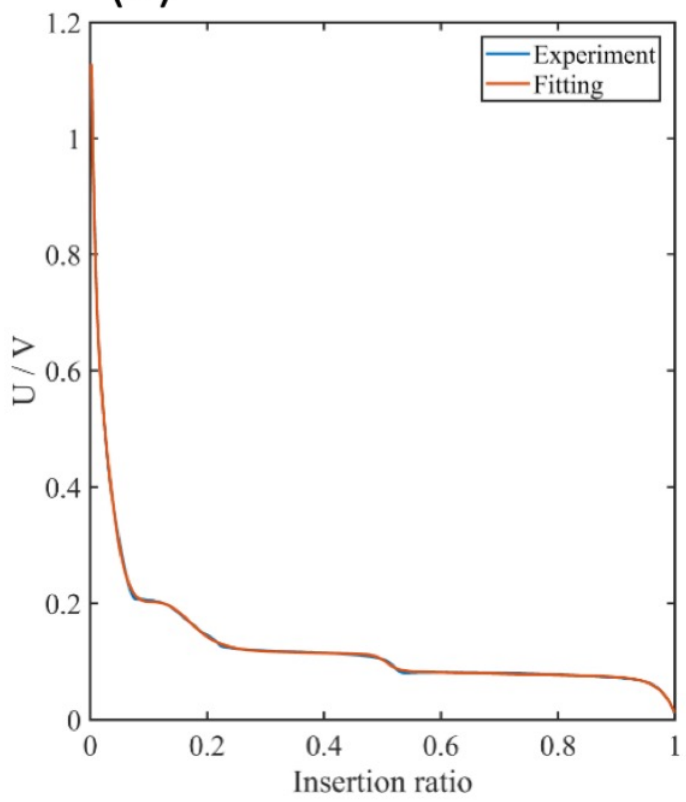

(c)

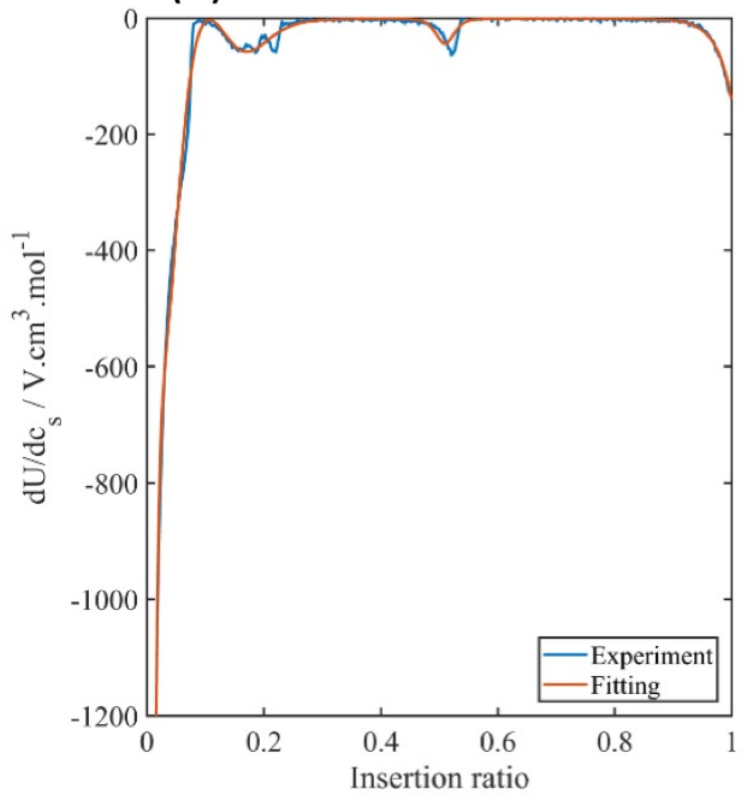

Figure 2 : (a) Evolution of the exchange current density with the SoC of the porous electrode. When the insertion ratio is equal to 0 , the porous electrode is considered to be fully delithiated. When the insertion ratio is equal to 1 , the porous electrode is considered to be fully lithiated. (b) Experimental charging curve achieved at a $C / 50$ rate on a 
graphite porous electrode and (c) the associated $\frac{\partial U}{\partial c_{s}}$ variation for a maximum concentration of lithium in the particle $c_{t}=16,110^{-3} \mathrm{~mol} . \mathrm{cm}^{-3}$.

Moreover, each SoC is also associated to a specific value of $\frac{\partial U}{\partial c_{S}}$ which can be determined from the charging curve of a porous electrode performed very low $C$-rate $(C / 50$ or $C / 20)$ to assume the steady state condition is achieved for each data point. This OCP curve can then be represented as a function of the insertion ratio $x$ as shown on Fig. $2 \mathrm{~b}$, similarly $\frac{\partial U}{\partial c_{S}}$ can be expressed as

$$
\frac{\partial U}{\partial c_{s}}=\frac{1}{c_{t}} \frac{\partial U}{\partial x}
$$

using Eq. 27 and calculated using the variation of the OCP with the insertion ratio and knowing $c_{t}$. Fig. 2c shows the variation of $\frac{\partial U}{\partial c_{s}}$ corresponding to the OCP curve presented in Fig. $2 \mathrm{~b}$.

Experimental OCP and $\frac{\partial U}{\partial c_{s}}$ curve were then fitted with a polynomial function to be used as input parameters for both the single particle and the porous electrode models.

\section{- Single Particle Model}

Using the experimental titration curve presented in Fig. $2 \mathrm{~b}$ for a graphite porous electrode, we first calculated the impedance $Z_{s p}$ of a single graphite particle at different SoC using a single set of parameters (Table I). For each impedance diagram depicted in Fig. 3a, different domains are observed: at high frequency, a semi-circle ascribed to the charge transfer at the electrode concomitant with the formation of the electrical double layer, and a $45^{\circ}$ slope line characteristic of the diffusion in the solid particles in the low frequency region. Note that a vertical line typical of a capacitive behavior is expected a very low frequency but is not observed in the frequency range explored. 


\begin{tabular}{ccc}
\hline \multicolumn{3}{c}{ Table I. Parameters used in the Single Particle } \\
Impedance simulations \\
\hline Parameter & Value & Reference \\
\hline$c_{t}$ & $16,1.10^{-3} \mathrm{~mol} . \mathrm{cm}^{-3}$ & [27] \\
$k$ & $10^{-4} \mathrm{~cm} \cdot \mathrm{s}^{-1}$ & Assumed \\
$F$ & $96485 \mathrm{C} \cdot \mathrm{mol}^{-1}$ & \\
$\alpha_{a}, \alpha_{c}$ & 0.5 & \\
$i_{0}$ & $F k^{\prime} c_{e}^{\alpha_{a}}(1-x)^{\alpha_{a} x^{\alpha}}$ & \\
$R_{s}$ & $1 \mu \mathrm{m}$ & [27] \\
$D_{s}$ & $2.10^{-12} \mathrm{~cm}^{2} \cdot \mathrm{s}^{-1}$ & [15] \\
$\frac{\partial U}{\partial c_{s}}$ & $-20.27 \mathrm{~V} \cdot \mathrm{cm}^{3} . \mathrm{mol}^{-1}$ & Assumed \\
$C_{d l}$ & $20 \mu \mathrm{Fm}^{-2}$ &
\end{tabular}

As the lithium content increases (i.e. when the SoC increases), the semi-circle diameter first decreases from $1 \%$ SoC to 50 \% SoC and then increases for higher SoC, following the evolution of the exchange current density (Fig 3.) as shown on Fig. 3a (charge transfer coefficient equal to 0.5). This specific behavior for SoC greater than $50 \%$, can be explained by the depletion of lithium hosting sites within the particle, which translates into charge transfer with higher impedance on the EIS diagrams. It is also worth mentioning some specific behaviors of the impedance diagrams:

- At $1 \%$ of SoC, the impedance response is characteristic of a capacitance behavior. Indeed, the porous electrode behaves as a blocking electrode resulting in an accumulation of charged species at the interface electrode/electrolyte. A similar behavior is observed at $100 \%$ of SoC (two top curves in Fig. 3a) because the porous electrode is fully lithiated. However, the difference observed on the simulated curves is ascribed to the value of $\frac{\partial U}{\partial c_{S}}$ (Fig. 2c)

- At $20 \%$ and $50 \%$ of SoC, the impedance response shows the charge transfer loop overlapping with the $45^{\circ}$ slope line characteristics of the diffusion in the solid particles. This unexpected behavior originates from a shift in the value of the diffusion part $Y_{S} \frac{\partial U}{\partial c_{S}}$ of 
the impedance solution given in Eq. 17 and more specifically of the parameter $\frac{\partial U}{\partial c_{s}}$ since $Y_{S}$ remains constant for any SoC at a given frequency. The fluctuations of $\frac{\partial U}{\partial c_{S}}$ at $20 \%$ and $50 \%$ of SoC as seen on the titration curve in Fig.2c correspond to phase transitions within the graphite particle (staging) which impact severely the impedance response.

(a)

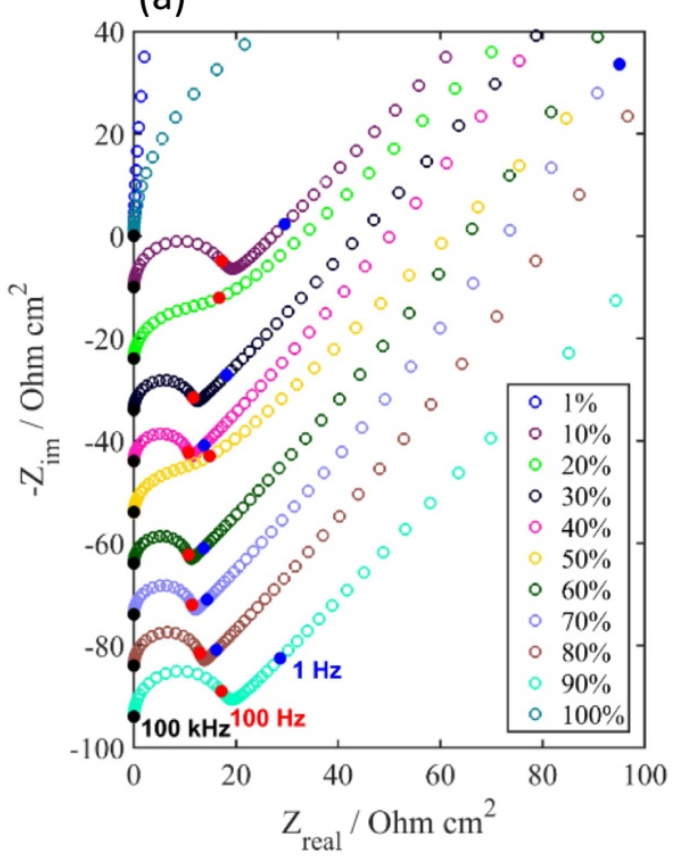

(b)

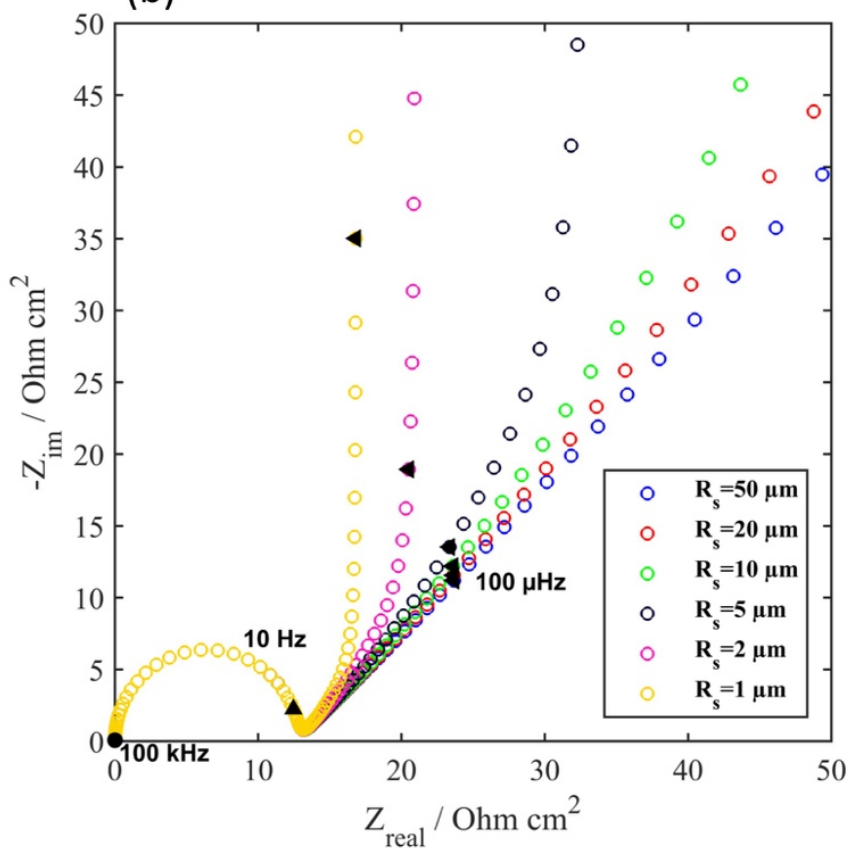

Figure 3: (a) Impedance simulations for a $1 \mu \mathrm{m}$ in diameter single graphite particle at different SoC showing the variation of the charge transfer with the lithiation degree (broadening of the low frequency semi loop). An offset has been added on the imaginary part of the impedance diagrams for the sake of clarity. (b) Impedance simulations of a single graphite particle as a function of the particle size for $60 \%$ of SoC. To better evidence the different time-constants, a diffusion coefficient in the solid phase, $D_{S}$, of $1.10^{-9} \mathrm{~cm}^{2} \mathrm{~s}^{-1}$ has been used.

The influence of the particle radius presented in Fig. 3b, shows that the low frequency capacitive behavior is observed at higher frequencies for particles with lower radius. This peculiar behavior can be interpreted in terms of penetration depth of the reaction. In other words, for large radius particles, the frequency perturbation is not low enough to affect the whole volume of the particle, i.e. all available hosting sites within the particle enable lithium diffusion (45 degree angle straight line). Conversely, the accumulation of lithium-ions inside the particle for smaller particles 
translates into a capacitive behavior (90 degree angle straight line) for the low frequencies presented in this work. It should be noted that the transition frequency between the diffusive behavior and the capacitive behavior depends on both the particle size and on the diffusion coefficient in the solid phase. For high frequencies, the charge transfer resistance remains constant and independent of the radius of particle because impedance is expressed per surface unit. For comparison, usual button battery results in impedance values spreading over few tens ohms from high to low frequencies.

\section{- Porous Electrode Model}

The parameters used for the simulations presented below are given Table II.

Fig. 4a shows a typical impedance diagram for a porous electrode composed of graphite particles. Compared to the single particle impedance simulations, a new domain appears in the middle to high frequency region. We indeed observe in addition to a non-perfect semi-circle, a $22.5^{\circ}$ slope characteristic of the diffusion of the electrolyte in the pore structure of the porous electrode [28, 29]. Interestingly, the semi-loop ascribed to the charge transfer in the high frequency region is also affected by the porous behavior of the system and shows a non-perfect semi-circle with an initial $45^{\circ}$ slope, as predicted by the work of de Levie [11]. 
a)

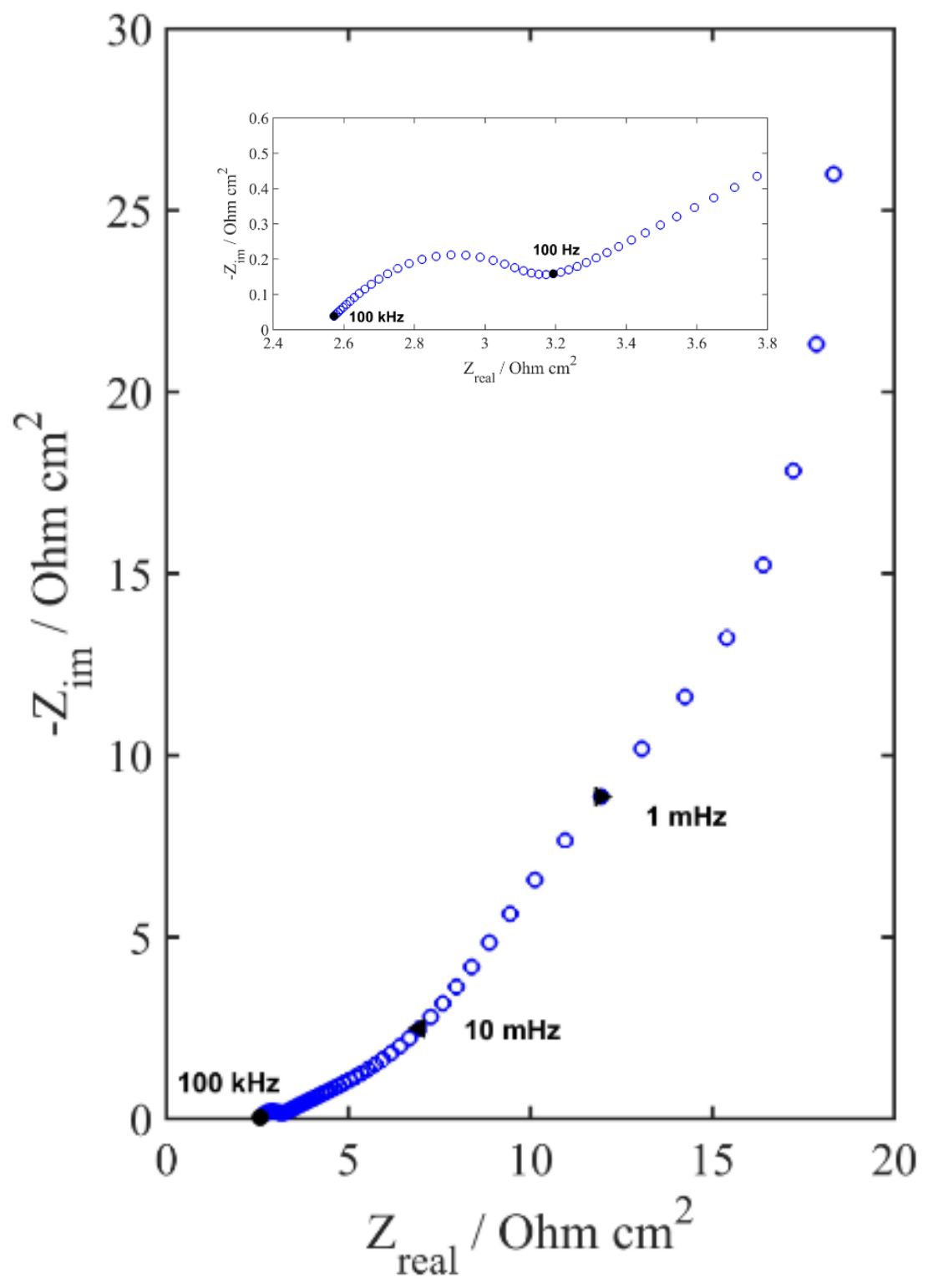




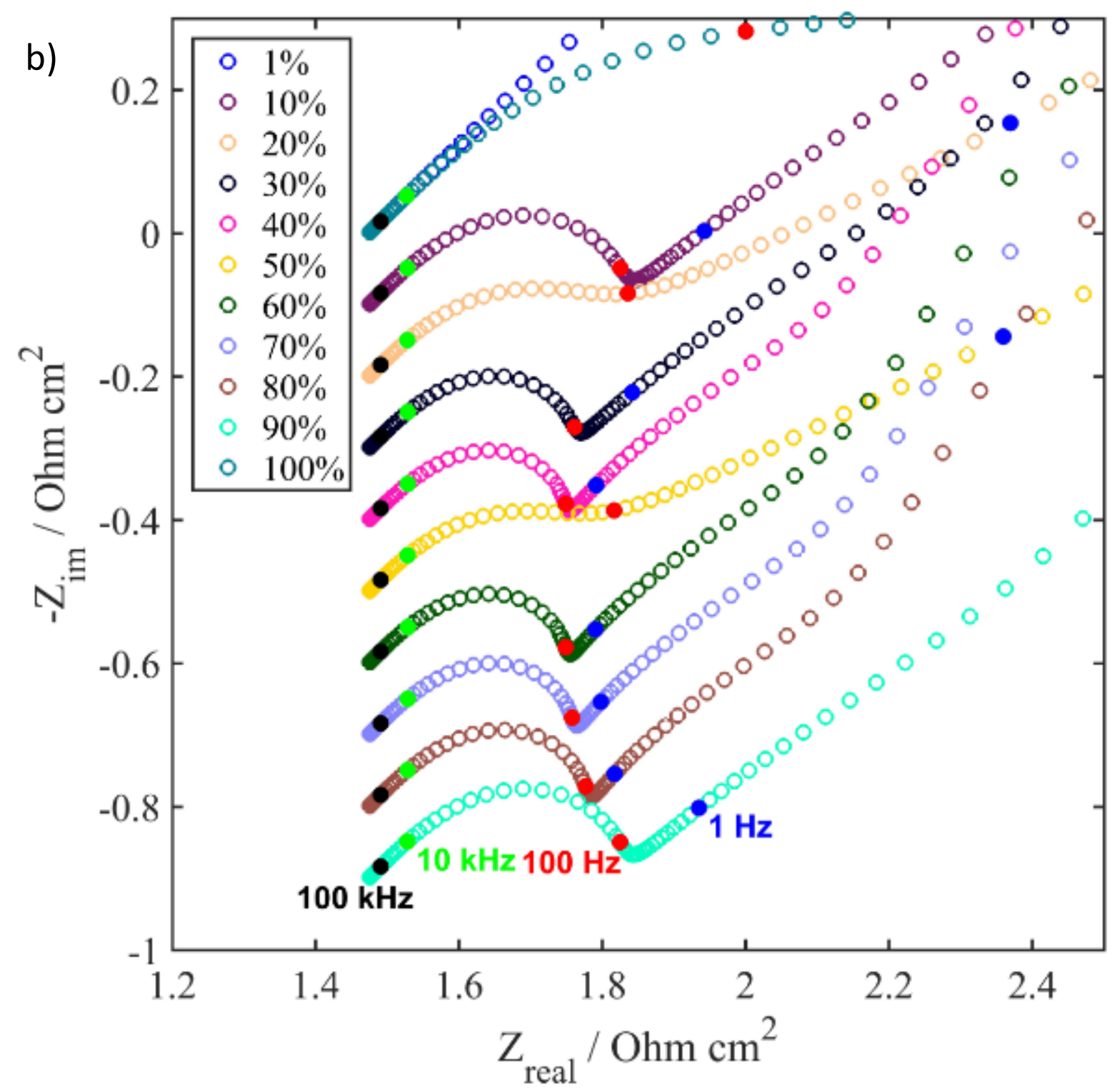

Figure 4: (a) Impedance simulations for a porous electrode composed of graphite particles in contact with the electrolyte considering an exchange current density $i_{0}=0.0036 \mathrm{A.cm} \mathrm{cm}^{-2}$ and $(b)$ influence of the state of charge of the electrode $(\mathrm{SoC})$-Simulation parameters are given in Table II. 


\begin{tabular}{|c|c|c|}
\hline Parameters & Value & Reference \\
\hline \multicolumn{3}{|c|}{ Geometrical parameters } \\
\hline$\delta$ & $100 \mu \mathrm{m}$ & [23] \\
\hline$\epsilon_{e}$ & 0.4 & [23] \\
\hline$\tau$ & $1-1.6 \ln \left(\epsilon_{e}\right)$ & [23] \\
\hline$l_{p}$ & $\delta . \tau$ & [23] \\
\hline$\epsilon_{s}$ & 0.2 & [23] \\
\hline$\epsilon_{b}$ & 0.05 & [23] \\
\hline$\epsilon_{a}$ & $1-\epsilon_{e}-\epsilon_{s}-\epsilon_{b}$ & [23] \\
\hline$a_{v}$ & $3 \frac{\epsilon_{a}}{R_{s}}$ & \\
\hline$R_{S}$ & $1 \mu m$ & [27] \\
\hline \multicolumn{3}{|c|}{ Transport and Kinetic parameters } \\
\hline$i_{0}$ & $F k^{\prime} c_{e}^{\alpha_{a}}(1-x)^{\alpha_{a}} x^{\alpha_{c}}$ & [27] \\
\hline$c_{t}$ & $16,1 \cdot 10^{-3} \mathrm{~mol} \cdot \mathrm{cm}^{-3}$ & [27] \\
\hline$k$ & $10^{-4} \mathrm{~cm} \cdot \mathrm{s}^{-1}$ & Assumed \\
\hline$F$ & 96485 C. $\mathrm{mol}^{-1}$ & \\
\hline$\alpha_{a}, \alpha_{c}$ & 0.5 & \\
\hline$D_{s}$ & $2.10^{-12} \mathrm{~cm}^{2} \cdot \mathrm{s}^{-1}$ & [27] \\
\hline$D_{e}$ & $2.6 \cdot 10^{-6} \mathrm{~cm}^{2} \cdot \mathrm{s}^{-1}$ & [27] \\
\hline$D_{e, e f f}$ & $D_{e} \cdot \frac{\epsilon_{e}}{\tau}$ & [23] \\
\hline$\kappa_{s}$ & $10.10^{-2} \mathrm{~S} . \mathrm{cm}^{-1}$ & [23] \\
\hline$\kappa_{s, e f f}$ & $\kappa_{s} \cdot \frac{\epsilon_{s}}{\tau}$ & [23] \\
\hline$\kappa_{e}$ & $10^{-2} \mathrm{~S} . \mathrm{cm}^{-1}$ & [23] \\
\hline$\kappa_{e, e f f}$ & $\kappa_{e} \cdot \frac{\epsilon_{e}}{\tau}$ & [23] \\
\hline$c_{e}$ & $1.10^{-3} \mathrm{~mol} . \mathrm{cm}^{-3}$ & \\
\hline$t^{+}$ & 0.363 & [27] \\
\hline$\frac{\partial U}{\partial c_{s}}$ & $-20.27 \mathrm{~V} \cdot \mathrm{cm}^{3} \cdot \mathrm{mol}^{-1}$ & [15] \\
\hline$f_{\bar{\mp}}$ & 1 & [27] \\
\hline$C_{d l}$ & $20 \mu \mathrm{F} . \mathrm{cm}^{-2}$ & \\
\hline
\end{tabular}


Fig. $4 \mathrm{~b}$ shows the evolution of the impedance of a graphite porous electrode as a function of the SoC. The effect of the polarization on porous electrode is quite similar to the one observed with the single particle, as the resistance to the charge transfer decreases from $1 \%$ to $50 \%$ of SoC and then increases for higher SoC due to the lack of available lithium hosting sites. We can also observe the same behavior as for the single graphite particles for $20 \%$ and $50 \%$ of SoC. For $1 \%$ SoC, the capacitive behavior is still observed but with a slope of $45^{\circ}$ due to the porosity of the electrode. For $100 \%$ SoC, the impedance is characterized by a very large charge transfer rate loop as observed for the single particle.

Fig. 5a shows the impedance diagrams corresponding to simulations for different particle size. For the set of results presented in this work, we consider that the porous electrode is constituted of identical particles. It can be seen from Fig. $5 b$ that changing the radius of the particle changes the charge transfer resistance. Indeed, the bigger the particles the larger the charge transfer loop. This result can be explain by the change of the specific interfacial area which is given by $a_{v}=$ $3 \frac{\epsilon_{a}}{R_{s}}$. As the volume fraction of active material is assumed to be constant, any increase of the particle radius reduces the active surface area resulting in an increase of the impedance and especially an increase of the charge transfer resistance. 
a)

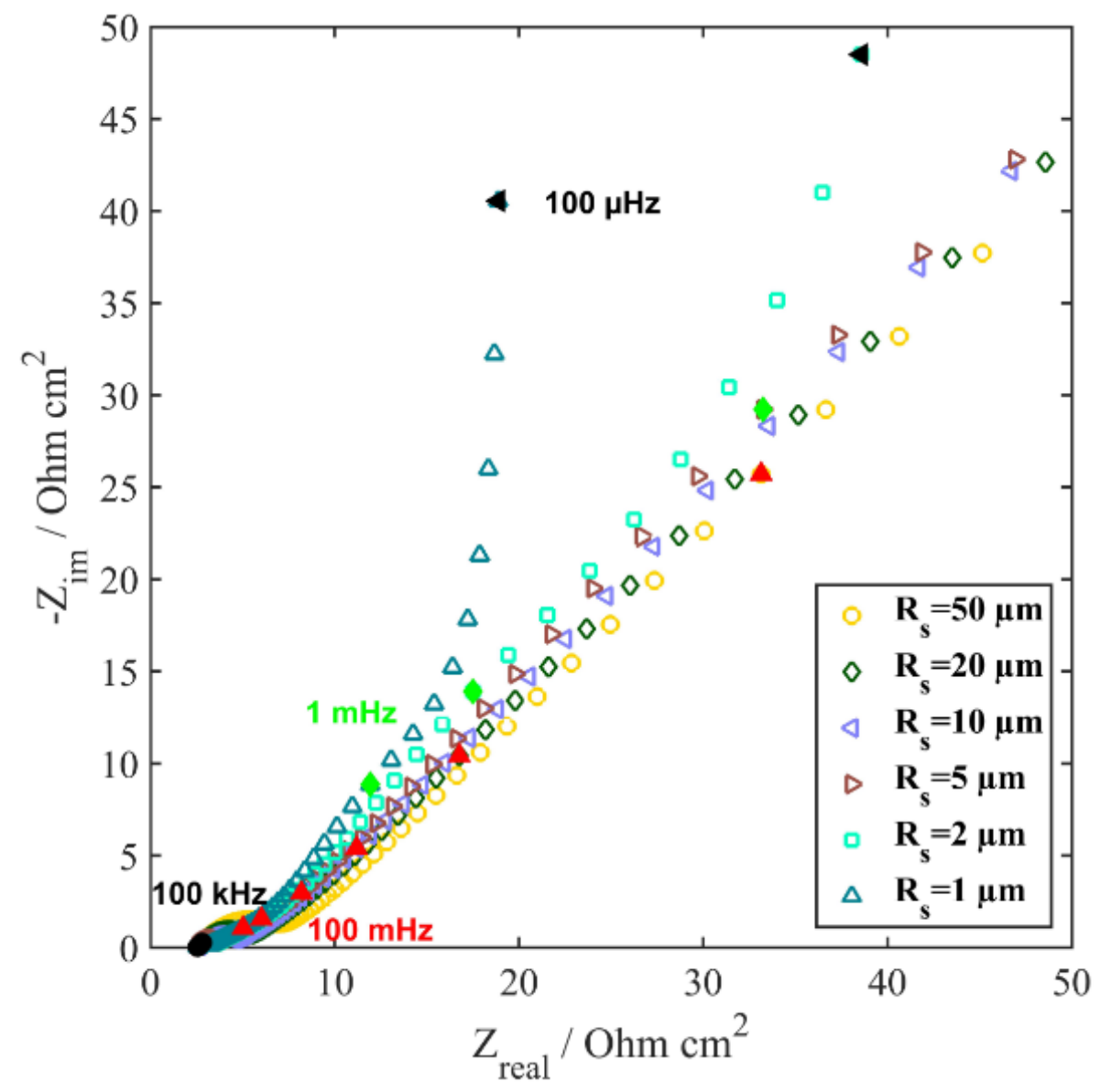

b)

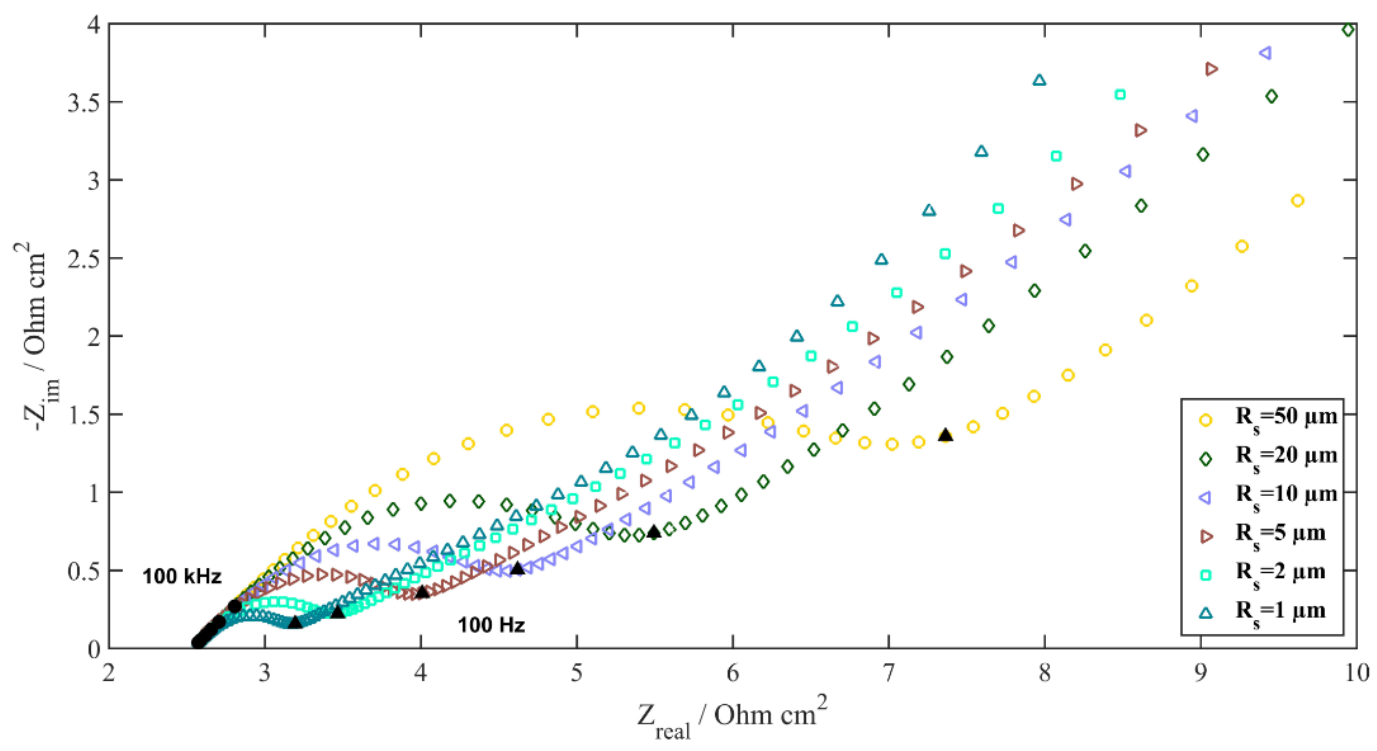

Figure 5: (a) Impedance simulations of a porous electrode composed of graphite particles in contact with the electrolyte for different particle size ( $R_{s}$ particle radius) considering an exchange current density $i_{0}=$ $0.0036 \mathrm{~A} \mathrm{~cm}^{-2}$; (b) zoom on the high frequency domain - Simulation parameters are given in Table II. 
We can observe that the change of the radius also influences the low frequency region corresponding to the diffusion of lithium in the solid phase, i.e. in the particles. The capacitive behavior is reached at higher frequency for smaller particles, which is in agreement with the single particle model previously discussed.

The influence of the thickness of the electrode, i.e. of the length of the pores, on the impedance response is presented in Fig. 6a. The frequency range associated to the diffusion in the pore clearly decreases when the thickness of the porous electrode is decreased. This can be explained from the model parameters since a decrease of the length of the electrode corresponds to a decrease of the length of the pore. In other words, the electrical signal penetrates the entire pore length at higher frequencies when the thickness decreases. These results also show that any change in the electrode thickness modifies the electrolyte resistance. Indeed, the electrolyte resistance measured in the high frequency domain is a characteristic of the electrode geometry and is independent of the electrochemical kinetics of the system under investigation. In this case, the electrolyte resistance is governed by both the electrode thickness and the pore resistance, which is given (for a single pore) by

$$
R_{\text {pore }}=\rho \frac{l_{p}}{S}
$$

where $\rho$ is the resistivity of the solution, $S$ is the section of the pore and $l_{p}$ is the length of a pore depending on the thickness of the electrode as shown in Table II. Eq. 30 shows that when the thickness of the electrode is increased, the pore resistance, and thus the electrolyte resistance, increase. From a practical point of view, distributions of pore length and pore section are expected and the impedance response reflects an average value for both parameters.

The variation of the electrolyte resistance with the thickness of the electrode can be seen on the analytical expression of the impedance of a porous electrode (Eq. 25), where the first term depends only on the length of the pore $l_{p}$ and the effective conductivity in the solid phase $\kappa_{s, e f f}$. 




(b)

(c)
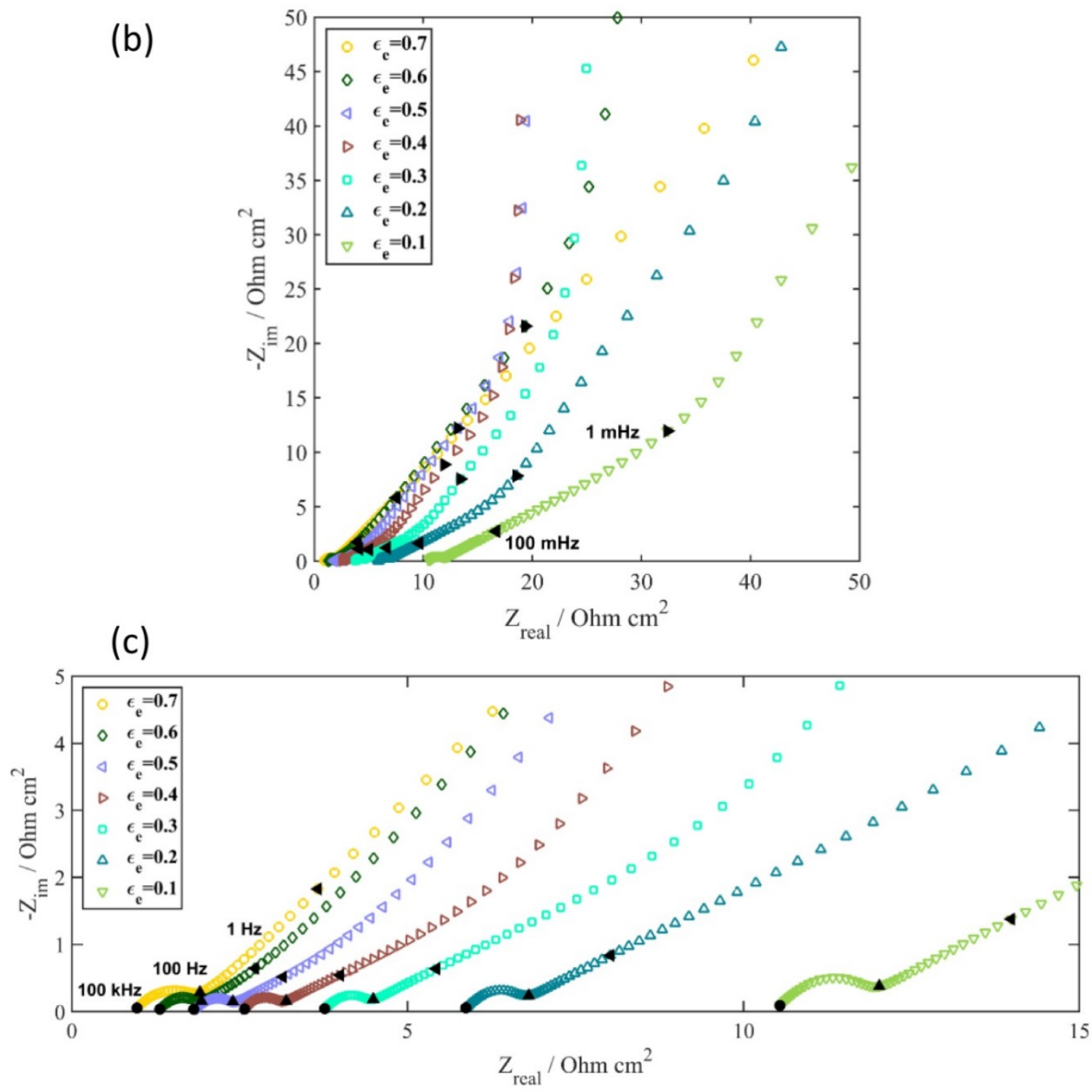
Figure 6: (a) Impedance simulations of a porous electrode composed of graphite particles as a function of the thickness of the porous electrode for $i_{0}=0.0036{\mathrm{~A} . \mathrm{cm}^{-2}}^{(b)}$ Influence of the porosity on the impedance simulations of a porous electrode composed of graphite particles for $i_{0}=0.0036 \mathrm{~A} \mathrm{~cm}^{-2}$; (c) zoom on the high frequency domain - Simulation parameters are given in Table II.

Fig. $6 \mathrm{~b}$ shows the variations of the impedance diagram as a function of the porosity of the electrode (from 0.1 to 0.7 ). The diffusion contribution in the pore of the electrode is less visible on the diagram when the porosity increases. Indeed, in Fig. 6b, the frequency domain associated to the diffusion in the pore has a lower impedance when the porosity of the porous electrode is larger. For low porosity - when the electrode is more compact - the diffusion inside the solutionphase becomes a limiting kinetic factor resulting as an increase in the impedance. By increasing the porosity, the pores fill with the electrolyte and the solution-phase diffusion is facilitated resulting in a decrease of the impedance. For a specific porosity of 0.6 , only the diffusion within the hosting particles is observed, the diffusion process inside the pore is therefore no longer a limiting kinetic process. This observation is however true for a given set of parameters (exchange current density, diffusion coefficient of lithium in the solution etc.), and the specific porosity described here changes with the parameters used for the simulation. As previously discussed, the porosity of the electrode strongly influence the electrolyte resistance (Fig. 6c).

Fig. 7a shows the evolution of the charge transfer resistance, determined from a graphical analysis of the impedance diagrams simulated as a function of the porosity of the electrode. Interestingly, the parabolic profile obtained shows that an optimal porosity for the porous electrode, minimizing the charge transfer resistance, can be predicted with from these simulations. 

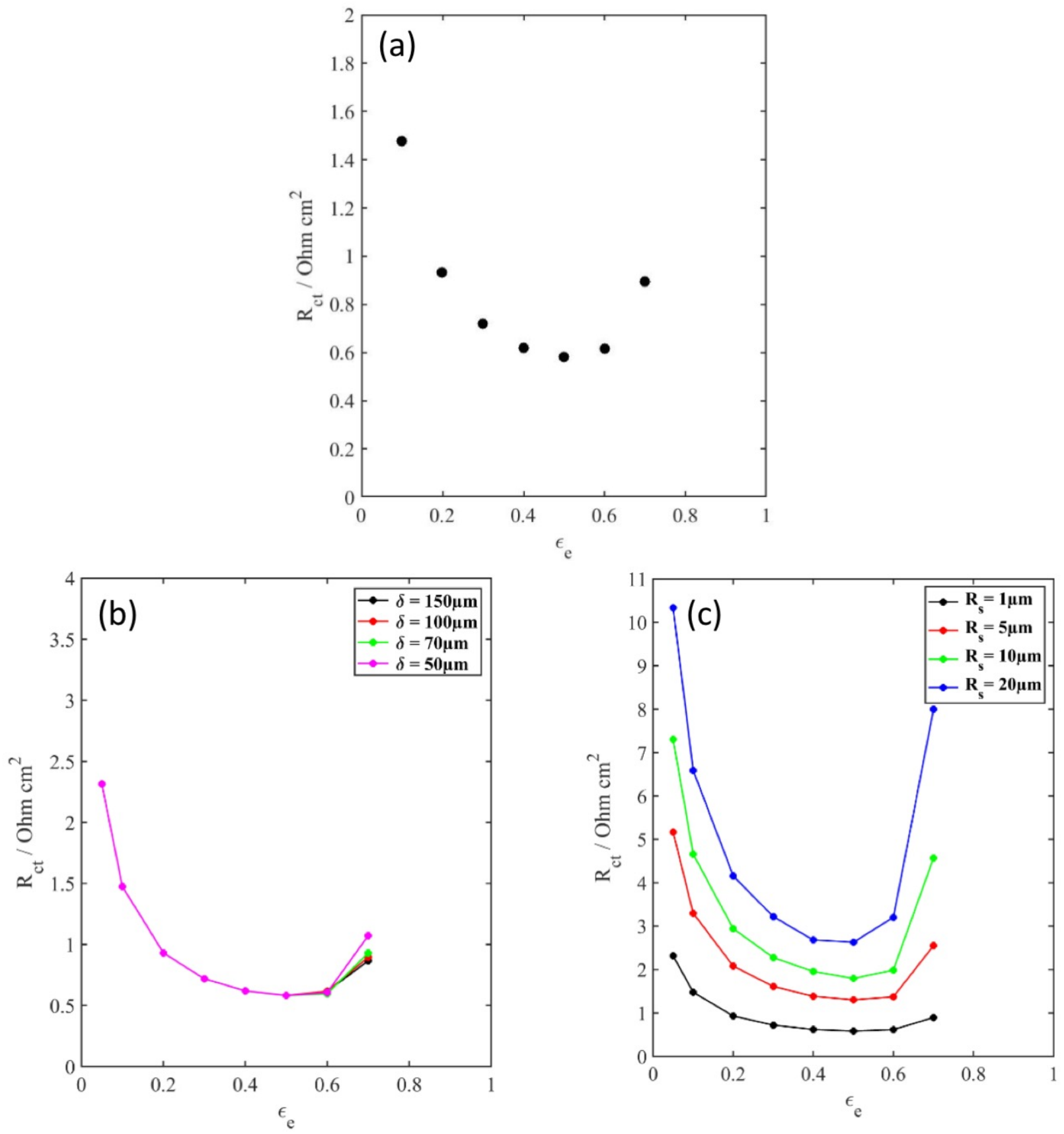

Figure 7: (a) Variation of the charge transfer resistance with the porosity of the electrode; (b) Evolution of the charge transfer resistance with the porosity for different electrode thickness; (c) and for different particle radius - Simulation parameters are given in Table II.

When the porosity diminishes, the effective conductivity of the solution-phase diminishes and thus the supply of lithium to the active material is lower. This results in an increase of the charge transfer resistance. On the other hand, increasing the porosity improves the effective solution- 
phase conductivity but also diminishes the volume fraction of active material. As a consequence, less host material is available for lithium, resulting in an increase of the charge transfer resistance. It is noteworthy that modifying the porosity of the electrode does not change the thickness of the electrode or the effective solid-phase conductivity. Indeed, we consider here the volume fraction of active material, binder and conductive carbon.

These simulations thus make possible to target a specific porosity depending on the kinetics and geometrical parameters of the system. Fig. 7b which shows the variations of the charge transfer resistance with the porosity of the electrode and for different thicknesses of the electrode, highlights that a similar parabolic profile is always observed. We can also notice that $R_{c t}$ value remains constant for all thickness except for porosity $\epsilon_{e}>0.6$. It also shows that for a high porosity, thin electrodes have a larger charge transfer rate than for thick electrodes. When increasing the porosity, the volume fraction of active material diminishes whereas the effective solution-phase conductivity remains the same. Thus, increasing the thickness of the electrode at constant porosity somehow promotes the contact between the active particles resulting in an easier charger transfer reaction. Interestingly, when increasing the radius of the particle, the charge transfer resistance increases, as shown in Fig. 7c. As explained before, this increase with the radius of the particle is ascribed to the decrease of the interfacial area. 

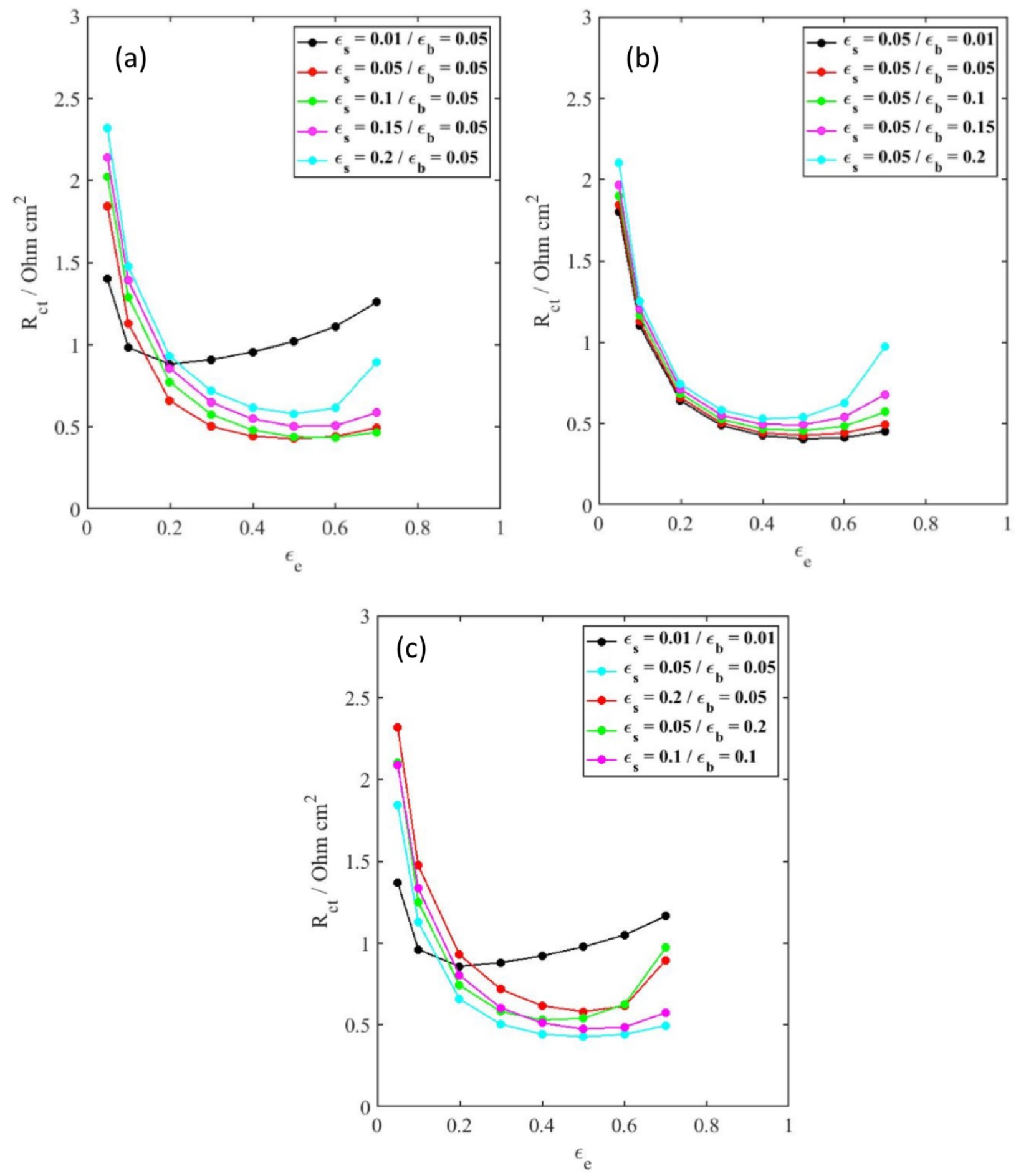

Figure 8: (a) Influence of the volume fraction of the conductive phase on the variation of the charge transfer resistance with the porosity; (b) influence of the volume fraction of the binder phase on the variation of the charge transfer resistance with the porosity; and (c) influence of the volume fraction of the conductive agent phase and the volume fraction of the binder phase on the variation of the charge transfer resistance with porosity - Simulation parameters are given in Table II. 
The influence of the radius of the particles is of both fundamental and practical interest since these results show the possibility to determine the optimal porosity of the electrode depending on its physical properties. Moreover, this optimal porosity can be determined as a function of the composition of the electrode that is a function of the amount of conductive agent, active materials and binder constituting the electrode. For a fixed value of the volume fraction of binder phase $\left(\epsilon_{b}=0.05\right)$ and varying the volume fraction of the conductive carbon phase $\epsilon_{s}$ (Fig. 8a), we observe that the ratio of conductive carbon influences the optimal porosity. For $\epsilon_{S}=0.01$, the charge transfer resistance is the largest because the effective solid-phase conductivity is very low. On the other hand, for high volume fraction of conductive carbon, the charge transfer resistance remains high. However, these results show that it is better to have too much conductive carbon in the electrode composition than not enough. The influence of the volume fraction of the binder phase induces less variations on the value of the optimal porosity as shown on Fig. 8b, for which $\epsilon_{s}$ was set to 0.05 and $\epsilon_{b}$ was varied. For higher binder content, the volume fraction of active material diminishes (the volume fractions of conductive carbon and solutionphase remain constant) resulting in a larger charge transfer resistance.

Fig. $8 \mathrm{c}$ shows the variation of the charge transfer resistance with the porosity for different ratio of conductive agent and binder phases. It can be seen that depending on the ratio of carbon black, active particles or binder, the optimal porosity varies.

Thus, the prediction of the impedance response of composite electrode allows the optimal porosity to be determined to achieve the fastest kinetics for lithium reaction. However, the model faces some limitations that need to be addressed:

- The analytical solution of the impedance used here is based on the Newman's model. So the impedance model has the same weaknesses than Newman's model beginning by the number of parameters involved. Indeed, the determination of some parameters is tedious experimentally and sometimes they are just assumed. Moreover, the high number of parameters facilitates the fitting of experimental impedance spectra. The interactions that might exist between particles are also not considered.

- This model does not take into account the principle of percolation. Even if we introduce the tortuosity which modifies the pore length, any change in the volume fraction of the 
conductive agent phase only modifies the conductivity of the solid matrix without considering the way the particles are organized. Indeed, it is assumed in this work that the local current is homogeneous all over phase the electrode which is not necessarily true.

\section{Conclusions}

In this study, a new insight is provided for the impedance modeling of porous electrodes describing battery electrode materials under operation. First, the common approximation made on the linearity of the Butler-Volmer's law for the calculation of the EIS diagram was not considered here, which permits to study the impedance as a function of the potential of the electrode, i.e. of the state of charge of the host material. Then, based on the pioneering work of Newman, and taking into account the model devised by Huang and Zhang, we developed and studied an analytical solution for the impedance of a single particle and for a porous electrode.

For the single particle model, we showed that the charge transfer resistance varies with the potential applied to the electrode and we studied the influence of active material particle size. We could establish a clear dependence between the value of the charge transfer resistance and the lithiation ratio of the particle. For the porous model, we investigated the influence of geometrical parameters of the electrode and we showed that it was possible to determine the porosity for which the charge transfer resistance is minimized and thus for which the kinetics of lithium reaction is optimal at a specific applied potential or current. We also showed that this optimal porosity strongly depends on the composition of the electrode, i.e. on the ratio of active material, conductive agent and binder.

\section{References}

1. Spahr, M.E., et al., Electrochemical insertion of lithium, sodium, and magnesium in molybdenum(VI) oxide. Journal of Power Sources, 1995. 54(2): p. 346-351.

2. Stevens, D.A. and J.R. Dahn, High capacity anode materials for rechargeable sodium-ion batteries. Journal of the Electrochemical Society, 2000. 147(4): p. 1271-1273.

3. Yabuuchi, N., et al., Research development on sodium-ion batteries. Chemical Reviews, 2014. 114(23): p. 11636-11682. 
4. Muldoon, J., C.B. Bucur, and T. Gregory, Quest for nonaqueous multivalent secondary batteries: Magnesium and beyond. Chemical Reviews, 2014. 114(23): p. 11683-11720.

5. Hong, S.Y., et al., Charge carriers in rechargeable batteries: Na ions vs. Li ions. Energy and Environmental Science, 2013. 6(7): p. 2067-2081.

6. Vivier, V., J. Farcy, and J.P. Pereira-Ramos, Electrochemical lithium insertion in sol-gel crystalline vanadium pentoxide thin films. Electrochimica Acta, 1998. 44(5): p. 831-839.

7. Aurbach, D., Review of selected electrode-solution interactions which determine the performance of $L i$ and Li ion batteries. Journal of Power Sources, 2000. 89(2): p. 206-218.

8. Rui, X.H., et al., Analysis of the chemical diffusion coefficient of lithium ions in Li3V2(PO4)3 cathode material. Electrochimica Acta, 2010. 55(7): p. 2384-2390.

9. Levi, M.D. and D. Aurbach, Simultaneous measurements and modeling of the electrochemical impedance and the cyclic voltammetric characteristics of graphite electrodes doped with lithium. Journal of Physical Chemistry B, 1997. 101(23): p. 4630-4640.

10. Gao, P., C. Zhang, and G. Wen, Equivalent circuit model analysis on electrochemical impedance spectroscopy of lithium metal batteries. Journal of Power Sources, 2015. 294: p. 67-74.

11. Levie, R.d., Electrochemical Response of Porous and Rough Electrodes. Adv. Electrochem. Electrochem. Eng 1967. 6: p. 329.

12. Lasia, A., IMPEDANCE OF POROUS-ELECTRODES. Journal of Electroanalytical Chemistry, 1995. 397(1-2): p. 27-33.

13. Keddam, M., C. Rakotomavo, and H. Takenouti, IMPEDANCE OF A POROUS-ELECTRODE WITH AN AXIAL GRADIENT OF CONCENTRATION. Journal of Applied Electrochemistry, 1984. 14(4): p. 437448.

14. Cachet, $\mathrm{C}$. and R. Wiart, COUPLED AXIAL GRADIENTS OF POTENTIAL AND CONCENTRATION IN A CYLINDRICAL PORE ELECTRODE - AN IMPEDANCE MODEL. Journal of Electroanalytical Chemistry, 1985. 195(1): p. 21-37.

15. Meyers, J.P., et al., The impedance response of a porous electrode composed of intercalation particles. Journal of the Electrochemical Society, 2000. 147(8): p. 2930-2940.

16. Doyle, M., J.P. Meyers, and J. Newman, Computer simulations of the impedance response of lithium rechargeable batteries. Journal of the Electrochemical Society, 2000. 147(1): p. 99-110.

17. Devan, S., V.R. Subramanian, and R.E. White, Analytical Solution for the Impedance of a Porous Electrode. Journal of The Electrochemical Society, 2004. 151(6): p. A905.

18. Sikha, G. and R.E. White, Analytical expression for the impedance response of an insertion electrode cell. Journal of the Electrochemical Society, 2007. 154(1): p. A43-A54.

19. Sikha, G. and R.E. White, Analytical Expression for the Impedance Response for a Lithium-lon Cell. Journal of the Electrochemical Society, 2008. 155(12): p. A893-A902.

20. Huang, J., et al., An Agglomerate Model for the Impedance of Secondary Particle in Lithium-Ion Battery Electrode. Journal of the Electrochemical Society, 2014. 161(8): p. E3202-E3215.

21. Dokko, K., et al., Kinetic characterization of single particles of LiCoO2 by AC impedance and potential step methods. Journal of the Electrochemical Society, 2001. 148(5): p. A422-A426.

22. Huang, J., et al., Analytical Solution to the Impedance of Electrode/Electrolyte Interface in LithiumIon Batteries. Journal of the Electrochemical Society, 2015. 162(13): p. A7037-A7048.

23. Huang, J. and J.B. Zhang, Theory of Impedance Response of Porous Electrodes: Simplifications, Inhomogeneities, Non-Stationarities and Applications. Journal of the Electrochemical Society, 2016. 163(9): p. A1983-A2000.

24. Doyle, M., Design and Simulation of Lithium Rechargeable Batteries (Ph. D Thesis). 1995, University of California at Berkeley: Lawrence Berkeley National Laboratory.

25. Newman, J. and K.E. Thomas-Alyea, Electrochemical Systems - Third Edition. John Wiley \& Sons, Inc., 2004. 
26. Fuller, T.F., M. Doyle, and J. Newman, Simulation and optimization of the dual lithium ion insertion cell. Journal of the Electrochemical Society, 1994. 141(1): p. 1-10.

27. Smith, K.A., C.D. Rahn, and C.-Y. Wang, Control oriented ID electrochemical model of lithium ion battery. Energy Conversion and Management, 2007. 48(9): p. 2565-2578.

28. Barcia, O.E., et al., Application of the impedance model of de Levie for the characterization of porous electrodes. Electrochimica Acta, 2002. 47(13-14): p. 2109-2116.

29. Barcia, O.E., et al., Further to the paper "Application of the impedance model of de Levie for the characterization of porous electrodes" by Barcia et al. [Electrochim. Acta 47 (2002) 2109]. Electrochimica Acta, 2006. 51(10): p. 2096-2097.

\section{Appendix 1: Derivation of the analytical expression of impedance of a porous}

\section{electrode}

Eq.23 and Eq.24 can be rewritten as

$$
\begin{gathered}
\frac{\partial^{2} \tilde{c}_{e}}{\partial x^{2}}=\theta_{1} \tilde{c}_{e}+\theta_{2}\left(\tilde{\phi}_{1}-\tilde{\phi}_{2}\right) \\
\frac{\partial^{2}\left(\tilde{\phi}_{1}-\tilde{\phi}_{2}\right)}{\partial x^{2}}=\theta_{3} \tilde{c}_{e}+\theta_{4}\left(\tilde{\phi}_{1}-\tilde{\phi}_{2}\right)
\end{gathered}
$$

where

$$
\begin{gathered}
\theta_{1}=\frac{j \omega \varepsilon}{D_{e, e f f}} \\
\theta_{2}=-\frac{1-t_{+}}{F D_{e, e f f}} \frac{a_{v}}{Z_{l o c}} \\
\theta_{3}=-\frac{2 j \omega \varepsilon R T\left(1-t_{+}\right)}{F D_{e, e f f} c_{0}}\left(1+\frac{\partial \ln f}{\partial \ln c_{e}}\right) \\
\theta_{4}=\left(\frac{2 R T\left(1-t_{+}\right)^{2}}{F^{2} D_{e, e f f} c_{0}}\left(1+\frac{\partial \ln f}{\partial \ln c_{e}}\right)+\left(\frac{1}{\kappa_{e, e f f}}+\frac{1}{\kappa_{s, e f f}}\right)\right) \frac{a_{v}}{Z_{l o c}}
\end{gathered}
$$


The two-equation system formed by Eq.26 and Eq.27 can then be expressed using matrix notation as

$$
\frac{\partial^{2}}{\partial x^{2}}\left[\begin{array}{c}
\tilde{c}_{e} \\
\tilde{\phi}_{1}-\tilde{\phi}_{2}
\end{array}\right]=\left[\begin{array}{c}
\theta_{1} \theta_{2} \\
\theta_{3} \theta_{4}
\end{array}\right]\left[\begin{array}{c}
\tilde{c}_{e} \\
\tilde{\phi}_{1}-\tilde{\phi}_{2}
\end{array}\right]
$$

From $X=\left(\begin{array}{c}\tilde{c}_{e} \\ \widetilde{\phi}_{1}-\widetilde{\phi}_{2}\end{array}\right)$ and $A=\left[\begin{array}{c}\theta_{1} \theta_{2} \\ \theta_{3} \theta_{4}\end{array}\right]$, we can come up to the following equation $\frac{\partial^{2}}{\partial x^{2}} X=A X$ The eigenvalues and the eigenvectors of this system are given by

$$
\begin{gathered}
\lambda_{1}=\frac{\theta_{1}+\theta_{4}-\sqrt{\left(\theta_{1}-\theta_{4}\right)^{2}+4 \theta_{2} \theta_{3}}}{2} \\
\lambda_{2}=\frac{\theta_{1}+\theta_{4}+\sqrt{\left(\theta_{1}-\theta_{4}\right)^{2}+4 \theta_{2} \theta_{3}}}{2} \\
V_{1}=\left(\begin{array}{c}
v_{1} \\
1
\end{array}\right)=\left(\begin{array}{c}
\frac{2 \theta_{2}}{\theta_{4}-\theta_{1}-\sqrt{\left(\theta_{1}-\theta_{4}\right)^{2}+4 \theta_{2} \theta_{3}}} \\
1
\end{array}\right) \\
V_{2}=\left(\begin{array}{c}
v_{2} \\
1
\end{array}\right)=\left(\begin{array}{c}
\frac{2 \theta_{2}}{\theta_{4}-\theta_{1}+\sqrt{\left(\theta_{1}-\theta_{4}\right)^{2}+4 \theta_{2} \theta_{3}}} \\
1
\end{array}\right)
\end{gathered}
$$

We then introduced the transformation matrix $P=\left[\begin{array}{cc}v_{1} & v_{2} \\ 1 & 1\end{array}\right]$, the diagonal matrix $H=\left[\begin{array}{cc}\lambda_{1} & 0 \\ 0 & \lambda_{2}\end{array}\right]$ and the vector $Y=\left(\begin{array}{l}y_{1} \\ y_{2}\end{array}\right)$.

Assuming

$$
X=P Y
$$

Then we can rewrite $\frac{\partial^{2}}{\partial x^{2}} X=A X$ as 


$$
\begin{gathered}
\frac{\partial^{2}}{\partial x^{2}} P Y=A P Y \\
\frac{\partial^{2}}{\partial x^{2}} Y=P^{-1} A P Y \\
\frac{\partial^{2}}{\partial x^{2}} Y=H Y
\end{gathered}
$$

Giving rise to the following system

$$
\left\{\begin{array}{l}
\frac{\partial^{2} y_{1}}{\partial x^{2}}=\lambda_{1} y_{1} \\
\frac{\partial^{2} y_{2}}{\partial x^{2}}=\lambda_{2} y_{2}
\end{array}\right.
$$

The solution to this system are

$$
\left\{\begin{array}{l}
y_{1}=\alpha_{1} \sinh \left(\sqrt{\lambda_{1}} x\right)+\alpha_{2} \cosh \left(\sqrt{\lambda_{1}} x\right) \\
y_{2}=\beta_{1} \sinh \left(\sqrt{\lambda_{2}} x\right)+\beta_{2} \cosh \left(\sqrt{\lambda_{2}} x\right)
\end{array}\right.
$$

From Eq.34, we can then express $\tilde{c}_{e}$ and $\tilde{\phi}_{1}-\tilde{\phi}_{2}$

$$
\begin{gathered}
\tilde{c}_{e}=v_{1}\left(\alpha_{1} \sinh \left(\sqrt{\lambda_{1}} x\right)+\alpha_{2} \cosh \left(\sqrt{\lambda_{1}} x\right)\right) \\
+v_{2}\left(\beta_{1} \sinh \left(\sqrt{\lambda_{2}} x\right)+\beta_{2} \cosh \left(\sqrt{\lambda_{2}} x\right)\right) \\
\tilde{\phi}_{1}-\tilde{\phi}_{2}=\left(\alpha_{1} \sinh \left(\sqrt{\lambda_{1}} x\right)+\alpha_{2} \cosh \left(\sqrt{\lambda_{1}} x\right)\right) \\
+\left(\beta_{1} \sinh \left(\sqrt{\lambda_{2}} x\right)+\beta_{2} \cosh \left(\sqrt{\lambda_{2}} x\right)\right)
\end{gathered}
$$

The constants $\alpha_{1}, \alpha_{2}, \beta_{1}$ et $\beta_{2}$ are obtained from the boundary conditions. 


$$
\begin{gathered}
{\left[\begin{array}{cccc}
\sqrt{\lambda_{1}} v_{1} & 0 & \sqrt{\lambda_{2}} v_{2} & 0 \\
\sqrt{\lambda_{1}} & 0 & \sqrt{\lambda_{2}} & 0 \\
\sinh \left(\sqrt{\lambda_{1}} l_{p}\right) v_{1} & \cosh \left(\sqrt{\lambda_{1}} l_{p}\right) v_{1} & \sinh \left(\sqrt{\lambda_{2}} l_{p}\right) v_{2} & \cosh \left(\sqrt{\lambda_{2}} l_{p}\right) v_{2} \\
c_{1} & c_{2} & c_{3} & c_{4}
\end{array}\right]} \\
\times\left[\begin{array}{l}
\alpha_{1} \\
\alpha_{2} \\
\beta_{1} \\
\beta_{2}
\end{array}\right]
\end{gathered}
$$

where

$$
\left[\begin{array}{l}
c_{1} \\
c_{2} \\
c_{3} \\
c_{4}
\end{array}\right]=\left[\begin{array}{l}
\sqrt{\lambda_{1}} \cosh \left(\sqrt{\lambda_{1}} l_{p}\right)\left(1-\frac{\theta_{3}}{\theta_{1}} v_{1}\right) \\
\sqrt{\lambda_{1}} \sinh \left(\sqrt{\lambda_{1}} l_{p}\right)\left(1-\frac{\theta_{3}}{\theta_{1}} v_{1}\right) \\
\sqrt{\lambda_{2}} \cosh \left(\sqrt{\lambda_{2}} l_{p}\right)\left(1-\frac{\theta_{3}}{\theta_{1}} v_{2}\right) \\
\sqrt{\lambda_{2}} \cosh \left(\sqrt{\lambda_{2}} l_{p}\right)\left(1-\frac{\theta_{3}}{\theta_{1}} v_{2}\right)
\end{array}\right]
$$

After calculation, we obtain

$$
\begin{gathered}
\alpha_{1}=-\frac{\tilde{l}_{a p p}}{\kappa_{s, e f f} \sqrt{\lambda_{1}}}\left(\frac{v_{2}}{v_{2}-v_{1}}\right) \\
\beta_{1}=\frac{\tilde{l}_{a p p}}{\kappa_{s, e f f} \sqrt{\lambda_{2}}}\left(\frac{v_{1}}{v_{2}-v_{1}}\right)
\end{gathered}
$$

$\alpha_{2}$

$=-\frac{\tilde{l}_{a p p}}{\kappa_{s, e f f}} \frac{1}{\sqrt{\lambda_{2}}\left(1-\frac{\theta_{3}}{\theta_{1}} v_{2}\right) v_{1} \tanh \left(\sqrt{\lambda_{2}} l_{p}\right)-\sqrt{\lambda_{1}}\left(1-\frac{\theta_{3}}{\theta_{1}} v_{1}\right) v_{2} \tanh \left(\sqrt{\lambda_{1}} l_{p}\right)}$ 


$$
\begin{aligned}
\left(-\frac{v_{2}}{\cosh \left(\sqrt{\lambda_{1}} l_{p}\right)}-\frac{\kappa_{e, e f f}}{\kappa_{s, e f f}} \frac{v_{2}}{v_{2}-v_{1}}\left(1-\frac{\theta_{3}}{\theta_{1}} v_{1}\right) v_{2}\right. \\
+\frac{\kappa_{e, e f f}}{\kappa_{s, e f f}} \frac{v_{2}}{v_{2}-v_{1}}\left(1-\frac{\theta_{3}}{\theta_{1}} v_{2}\right) v_{1}\left(\frac{1}{\cosh \left(\sqrt{\lambda_{1}} l_{p}\right) \cosh \left(\sqrt{\lambda_{2}} l_{p}\right)}\right. \\
\left.\left.+\frac{\sqrt{\lambda_{2}}}{\sqrt{\lambda_{1}}} \tanh \left(\sqrt{\lambda_{1}} l_{p}\right) \tanh \left(\sqrt{\lambda_{2}} l_{p}\right)\right)\right)
\end{aligned}
$$

$\beta_{2}$

$$
\begin{gathered}
=-\frac{\tilde{l}_{a p p}}{\kappa_{s, e f f}} \frac{1}{\sqrt{\lambda_{2}}\left(1-\frac{\theta_{3}}{\theta_{1}} v_{2}\right) v_{1} \tanh \left(\sqrt{\lambda_{2}} l_{p}\right)-\sqrt{\lambda_{1}}\left(1-\frac{\theta_{3}}{\theta_{1}} v_{1}\right) v_{2} \tanh \left(\sqrt{\lambda_{1}} l_{p}\right)} \\
\left(\frac{v_{1}}{\cosh \left(\sqrt{\lambda_{2}} l_{p}\right)}-\frac{\kappa_{e, e f f}}{\kappa_{s, e f f}} \frac{v_{1}}{v_{2}-v_{1}}\left(1-\frac{\theta_{3}}{\theta_{1}} v_{2}\right) v_{1}\right. \\
+\frac{\kappa_{e, e f f}}{\kappa_{s, e f f}} \frac{v_{1}}{v_{2}-v_{1}}\left(1-\frac{\theta_{3}}{\theta_{1}} v_{1}\right) v_{2}\left(\frac{1}{\cosh \left(\sqrt{\lambda_{1}} l_{p}\right) \cosh \left(\sqrt{\lambda_{2}} l_{p}\right)}\right. \\
\left.\left.+\frac{\sqrt{\lambda_{1}}}{\sqrt{\lambda_{2}}} \tanh \left(\sqrt{\lambda_{1}} l_{p}\right) \tanh \left(\sqrt{\lambda_{2}} l_{p}\right)\right)\right)
\end{gathered}
$$

When expressing the impedance $Z_{p e}=\frac{\widetilde{\Phi}_{1}(x=0)-\widetilde{\Phi}_{2}\left(x=l_{p}\right)}{\tilde{i}_{a p p}}$, the term $\Lambda$ appears



